\title{
Cierre de Escuelas y Desigualdad Socioeducativa en Tiempos del Covid-19. Una Investigación Exploratoria en Clave Internacional
}

\author{
School Closings and Socio-Educational Inequality in Times of \\ Covid-19. An Exploratory Research in an International Key
}

\author{
Jorge Cáceres-Muñoz * \\ Antonio Salvador Jiménez Hernández \\ Miguel Martín-Sánchez \\ Universidad de Extremadura, España
}

\begin{abstract}
La pandemia del Covid-19, causada por el virus SARS-CoV-2, ha cambiado el modus vivendi de miles de personas en todo el mundo. La excepcionalidad y la emergencia ha influido en diversos sectores y ámbitos de la vida social e institucional de los seres humanos como el sanitario, económico, político o educativo. El objetivo de este estudio es ofrecer una perspectiva internacional sobre el proceso de cierre de escuelas y sus consecuencias educativo-sociales a través de las percepciones de informantes clave. Con una muestra que alcanza 23 países y 4 continentes distintos, las percepciones se han analizado en base a siete categorías: Tiempos en la ejecución, anticipación o demora de medidas. Carácter democrático-participativo de las medidas educativas tomadas. Adaptación curricular, didáctica y metodológica. Infraestructura y recursos para una adecuada formación a distancia. Percepciones acerca del profesorado. Percepciones sobre el entorno familiar. Propuestas alternativas. Los resultados muestran una elevada diversidad de maneras de afrontar esta situación, pero con similares consecuencias para la población más vulnerable. Ello implica una llamada de atención a responsables y agentes del ámbito educativo para frenar la desigualdad educativa y social que el Covid-19 puede estar generando.
\end{abstract}

Descriptores: Educación; Covid-19; Cierre de escuelas; Desigualdad social.

The Covid-19 pandemic, caused by the SARS-CoV-2 virus, has changed the modus vivendi of thousands of people around the world. Exceptionality and emergency have influenced various sectors and areas of the social and institutional life of human beings, such as health, economics, politics or education. The objective of this study is to offer an international perspective on the process of school closure and its educational-social consequences through the perceptions of key informants. With a sample that reaches 23 countries and 4 different continents, perceptions have been analyzed based on seven categories: Timing of execution, anticipation or delay of measures. Democratic-participatory nature of the educational measures taken. Curricular, didactic and methodological adaptation. Infrastructure and resources for adequate distance training. Perceptions about teachers. Perceptions of the family environment. Alternative proposals. The results show a high diversity of ways of facing this situation, but with similar consequences for the most vulnerable population. This implies a wake-up call to managers and agents in the educational field to curb the educational and social inequality that Covid-19 may be generating.

Keywords: Education; Covid-19; School closings; Social inequality.

*Contacto: jorgecm@unex.es

ISSN: 2254-3139

www.rinace.net/riejs/

revistas.uam.es/riejs
Recibido: 20 de mayo 2020

$1^{\text {a }}$ Evaluación: 17 de junio 2020

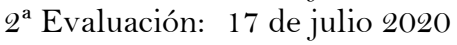

Aceptado: 16 de julio 2020 


\section{Introducción}

El brote de Covid-19, causado por el virus SARS-CoV-2 con origen en la ciudad de Wuhan, provincia china de Hubei, ha desencadenado desde diciembre de 2019 una pandemia mundial con consecuencias extraordinariamente difíciles para todos los sectores, con especial problemática para el sanitario y el económico (McKibbin y Fernando, 2020).

La virulencia de este fenómeno, ya mundial, hace que el reto se acreciente más si cabe en los casos con mayor grado de vulnerabilidad. Hablamos de personas con bajos recursos económicos, incluso sin hogar, aquellas que no disponen de empleo o que lo han perdido durante la pandemia, comunidades excluidas de manera regular, el colectivo migrante, personas con diversidad funcional, etc. (Berger et al., 2020). Ofrecer una respuesta adecuada requerirá un esfuerzo político y social a gran escala y multinivel para paliar situaciones complejas y de acuciante desigualdad. El Covid-19 va a evaluar la capacidad de reacción de los países desde una perspectiva nacional, pero también la cooperación desde una perspectiva internacional. La cohesión social va a estar determinada por la respuesta a la presión de los sistemas de protección social basados en la trayectoria de las decisiones de la política de cada país y la capacidad de actuar de manera responsable y generosa del ente macropolítico y macroeconómico actual (Dayrit y Mendoza, 2020). En el caso de la Unión Europea, ésta se enfrenta a un gran reto teniendo que ofrecer una respuesta de mayor coordinación y eficacia que la demostrada ante anteriores fenómenos coyunturales como la crisis financiera mundial de 2008 o la crisis migratoria irregular que está presente en el continente desde 2015. La colaboración a nivel mundial entre grandes instituciones como Naciones Unidas, el G20, la OMS, el Fondo Monetario Internacional, la Unión Africana y otras autoridades de terceros países será crucial (Jones et al., 2020). Por otro lado, en zonas geográficas que en nada tienen que ver con lo que ocurre en el primer mundo, el Covid-19 puede llegar a generar consecuencias fatales. Son los casos de ciertos países de África (Gilbert et al., 2020) y América Latina y Caribe (CEPAL, 2020). Una respuesta precoz por parte de sus gobernantes al igual que una ayuda externa coordinada, permitirían paliar escenarios de pobreza y subdesarrollo acrecentados.

Ante todas estas circunstancias, el derecho a una educación basada en criterios mínimos de igualdad y calidad para todos y todas puede verse fuertemente perjudicado. Ante esta situación, desde el punto de vista social y educativo, surgen una serie de preguntas en relación con el rendimiento educativo durante la pandemia, o lo referente a mantener activos los valores y principios pedagógicos y sociales de la escuela inclusiva, o la siempre necesidad de reivindicar, desde una posición crítica y activa pedagógica y políticamente hablando, de la exigencia moral y como sociedad de garantizar la equidad educativa.

Las consecuencias del Covid-19 atentan directamente contra el derecho a la educación. Y es que no podemos olvidar que el derecho a la educación no es solo garantizar el acceso a la instrucción (ahora online), sino el derecho a una educación de calidad, con procesos inclusivos que garanticen la igualdad de oportunidades real y no solo formal, porque la igualdad real potencia la justicia social (García-Gómez, 2018).

A pesar de la gran producción, análisis, información, e infoxicación sobre el COVD-19 en estos tiempos, desde un punto de vista formal y científico, responder a los interrogantes antes planteados no es fácil, ni sencillo ni tampoco breve. 
Algunas entidades de solvencia científica reconocida, como la UNESCO, se han aventurado a realizar algunas aproximaciones a la situación y consecuencias que la pandemia está generando en la población escolar. Recientemente anunció que los cierres de escuelas a nivel nacional estarían afectando al 90\% de la población estudiantil de todo el mundo. Desde un análisis inicial señala varios puntos perjudiciales como consecuencia de este cierre: la interrupción del aprendizaje, el no acceso a una alimentación regular, la falta de competencia parental y recursos de las familias para adaptarse a una situación de escolarización a distancia, el acceso desigual a la repentina digitalización educativa, insuficiencias en materia de cuidados a la infancia por imposibilidad de conciliación familiar, pérdida de músculo económico intrafamiliar, aumento de la presión en instituciones educativas que sí permanecen abiertas y tendencias crecientes en las tasas de abandono escolar ${ }^{1}$. Todas estas circunstancias se ven incrementadas para sectores de la población con menos recursos, como señalábamos en líneas anteriores, con mayores dosis de vulnerabilidad. En este sentido, en España, uno de los países más afectados por la pandemia en número de contagios y fallecimientos ${ }^{2}$ cabe mencionar con especial énfasis la situación de vulnerabilidad a la que puede estar sometida la infancia y la juventud. Como muestran los datos del observatorio Social La Caixa (2020), la pobreza infantil y juvenil en 2017 se encontraba varios puntos por encima de la media europea, pudiéndose incrementar todavía más esta desigualdad a causa de la crisis sanitaria provocada por el Covid-19.

En definitiva, la fortaleza o fragilidad de los sistemas educativos y la naturaleza de las medidas educativas aplicadas ante esta crisis no ha sido la misma en todos los países, lo que hace más complejo ofrecer una mirada clara de la situación mundial actual. Ante esta incertidumbre y la multiplicidad de frentes abiertos solo en el terreno educativo, este estudio pretende contribuir a alcanzar mayor claridad sobre esta situación.

El objetivo de este estudio es, a través de una investigación exploratoria, ofrecer una perspectiva internacional sobre el proceso de cierre de escuelas por el Covid-19 y sus posibles consecuencias educativo-sociales a través de las percepciones de informantes clave. En las próximas páginas se realizará, en primer lugar, una revisión de la literatura acerca del impacto educativo que puede estar generando ya la crisis sanitaria que servirá igualmente como justificación del trabajo realizado. En segundo lugar, se presentará la metodología de estudio y las fases ejecutadas. Por último, se muestran y analizan los resultados y su discusión en base a una serie de categorías cruciales que tratarán de analizar los factores que pueden estar generando desigualdades en el terreno educativo y social, terminando con unas propuestas de acción planteadas por los expertos consultados en cada país.

\footnotetext{
${ }^{1}$ UNESCO. Consecuencias negativas del cierre de las escuelas. https://es.unesco.org/covid19/educationresponse/consecuencias

${ }^{2}$ Ministerio de Sanidad. Gobierno de España. Enfermedad por nuevo coronavirus, Covid-19-Información actualizada sobre el brote.

https://www.mscbs.gob.es/profesionales/saludPublica/ccayes/alertasActual/nCov-

China/situacionActual.htm
} 


\section{Revisión de la literatura}

Como se trata de un trabajo científico, y no de una tertulia televisa, no nos atrevemos a conjeturar o a profetizar el fin de la escuela tal y como la conocemos, porque eso, en nuestra opinión, es muy complicado. Si algo ha demostrado el sistema educativo y la escuela como institución es que, a pesar de las grandes crisis que han azotado a la humanidad en los últimos siglos, la escuela ha permanecido prácticamente inamovible en sus bases, hierática y con aroma decimonónico. Sin embargo, algo sí se ha movido, levemente. La capacidad de los actores de las instituciones educativas, los docentes, los equipos directivos y las administraciones educativas para hacer frente, de un día para otro, a las consecuencias del cierre de escuelas, así como del teletrabajo, es una muestra de estos cambios. También ha puesto al límite al alumnado y sus familias, quienes tienen que nadar en aguas desconocidas y aprender a una gran velocidad nuevas competencias que hasta hace unos meses estaban proyectadas para un futuro no tan cercano.

Esa inmediatez de la realidad que se vive actualmente aún no ha generado el suficiente número de estudios comparados a nivel internacional sobre el cierre de las escuelas que puedan sostener un estado de la cuestión al que estaríamos acostumbrados. Sin embargo, aún sin establecer comparativas y siendo más bien específicos y locales, sí existen estudios sólidos sobre las consecuencias que está generando para la ciudadanía las adaptaciones a una vida educativa nueva, fruto de las medidas tomadas por la pandemia generada por el Covid-19.

Ante esto, este estudio siempre tendrá como objetivo preferente hallar la posible desigualdad educativo social presente y latente por esta crisis. Esto nos lleva a asumir como norma el no dejar pasar desapercibido lo que está ocurriendo con el alumnado más vulnerable, ese grupo social caracterizado porque en condiciones de escolarización habitual presenta dificultades de aprendizaje, que convive con circunstancias adversas en su entorno familiar, ya sea por razón económica o por déficit en las relaciones intrafamiliares, y aquel que manifiesta una diversidad funcional cuya adaptación para esta nueva eventualidad presenta un alto coste (Jiménez, 2020). Si la educación busca el desarrollo integral de los sujetos, nos preguntamos si en los tiempos del Covid-19 existen las mismas oportunidades para todos o si alguien se está quedando atrás.

La situación educativa previa a la aparición del Covid-19 estaba representada bajo una dicotomía en la que, por un lado, se establecían ciertas aspiraciones para mejorar el acceso y la calidad de la educación en los sistemas educativos mundiales, y por otro, la incapacidad para acometer ciertas deficiencias endémicas que no acababan de encontrar solución (universalización de la educación infantil, escolarización plena en educación primaria, elevación del grado de adquisición de competencias, dotación de recursos para el acceso a una educación de calidad en los países más pobres). Ello puede apreciarse en la trayectoria de compromisos acordados desde 1990 en Jomtiem con la Declaración Mundial sobre Educación para todos (UNESCO, 1990), o con los Marcos de Acción Regionales de África Subsahariana, Américas, Países Árabes, Asia y el Pacífico, Europa y América del Norte y países del Grupo E-9 (UNESCO, 2000) donde son ratificados los anteriores con vistas a cumplir objetivos en 2015. Iniciativas como estas, sufrirían actualizaciones y especificaciones como por ejemplo con las metas propuestas en materia educativa en la Estrategia Europa 2020 (Arriazu y Solari, 2015) y actualmente, con la aspiración de alcanzar el denominado objetivo 4: Garantizar una educación inclusiva y equitativa de calidad 
y promover oportunidades de aprendizaje permanente para todos, en el marco de los objetivos de Desarrollo sostenible para la agenda 2030 de Naciones Unidas (ONU, 2017).

Todos estos compromisos basados en el horizonte de la educación y que son previos a la aparición del Covid-19 mostraban la hoja de ruta internacional en la que los idearios económicos y sociales de las dos primeras décadas del siglo XXI se ven representados condicionando las agendas educativas; marcados por supuesto, por las coyunturas económicas derivadas de la crisis de 2008, la globalización y la transformación social y tecnológica provocada por el desarrollo veloz de las Tecnologías de la Información y la Comunicación. Hoy, a esa enumeración de elementos se suma la crisis sanitaria mundial, obligando a paralizar y puede que a rediseñar una vez más las agendas educativas, y por ende, a evitar el crecimiento de la brecha entre diversos colectivos que ven como la desigualdad y la distancia con los que deberían ser sus iguales se ve ampliada.

El debate es constante en relación con las medidas que se están tomando desde distintos sectores y que las administraciones de cada país, estado o región ponen en marcha. Muchos países basan sus medidas en las acciones que se llevaron a cabo para combatir el SARS en 2003 (Ting et al., 2020). El debate político, la opinión pública, la prensa, las redes sociales, en todos los espacios hay argumentaciones a favor y en contra de las estrategias para hacer frente al virus. Dentro del ámbito educativo el debate también está presente. En el seno de la comunidad científica existen trabajos que se muestran a favor del cierre de las escuelas para el frenado de los contagios (Viner et al., 2020). Por otro lado, el debate también centra la idea acerca de cómo debería ser el proceso de vuelta a la normalidad. Ya hay estudios que señalan la evidencia sustancial de que la transmisión volvió a surgir en cierta medida por ejemplo en Hong Kong, una vez que las escuelas volvieron a abrir, protagonizándose episodios con falta de consenso acerca de los tiempos de cierre y reapertura de las escuelas (Cowling et al., 2020). En la reapertura, las decisiones políticas apoyadas y refrendadas por los técnicos deberán estar marcadas por la prudencia y el análisis certero de la realidad. En este debate se entremezcla la opinión política, la técnica y la del poder de la prensa, quién ejerce de canal de información para la masa ciudadana que vive a expensas de las decisiones que sus gobernantes tomen ${ }^{3}$. Ante este cúmulo de pareceres hay otros que se muestran prudentes o incluso dubitativos ante el cierre drástico de las escuelas que en muchos países ha tenido lugar. Se advierte una necesaria reflexión acerca de lo que supone el cierre en materia social y económica y si compensa, teniendo en cuenta la naturaleza de baja sintomatología y mortalidad del Covid-19 en la población infantil (Armitage y Nellums, 2020). Los autores señalan con vehemencia las problemáticas que la infancia más vulnerable puede estar sufriendo ante la imposibilidad de acudir a una escuela presencialmente, una escuela en donde sentirse protegido. Los contextos mediados por un acceso a la educación a distancia a través de tecnologías digitales se antojan desiguales, lo que requiere un proceso evaluativo de las acciones

\footnotetext{
${ }^{3}$ El País (España). La crisis del coronavirus. https://elpais.com/sociedad/2020-05-04/el-gobierno-planealimitar-a-15-el-numero-de-alumnos-por-aula-el-curso-que-viene.html. ABC (España). Sociedad.

https://www.abc.es/sociedad/abci-celaa-dice-solo-mitad-alumnos-volvera-clase-septiembre-si-no-vacuna202005042038_noticia.html. Le Monde (Francia). Société Éducation.

https://www.lemonde.fr/societe/article/2020/05/05/dans-les-ecoles-primaires-veillee-d-armes-avant-ledeconfinement_6038658_3224.html. Bild (Alemania). Corona-Krise.

https://www.bild.de/politik/inland/politik-inland/coronavirus-im-mai-koennten-die-ersten-schulenwieder-oeffnen-sagt-der-aerzte-ch-69718338.bild.htm. El Mercurio (Chile). Educación. https://www.emol.com/educacion/
} 
implementadas, sobre todo en contextos de mayor vulnerabilidad (Cervantes Holguín y Gutiérrez Sandoval, 2020).

Desde el punto de vista social y de la conciliación laboral, el cierre de comedores escolares suscita muchos problemas a los familiares y los padres que siguen trabajando de manera presencial; a menudo pueden estar obligados a dejar a los niños sin supervisión o bien renunciar a los empleos con la consecuencia económica que ello comporta. A tenor de ello, Álvarez Zarzuelo (2020) presenta una serie de necesidades que merecen atención y que son susceptibles de aparecer en múltiples hogares aislados durante esta crisis. Por ejemplo, aquellas que tienen que ver con la sensación de soledad derivada de una imposibilidad de acceso a internet, o aquellos que viven en contextos donde se desarrollan episodios de violencia intrafamiliar y violencia machista; aquellos niños y niñas que pueden sufrir abuso sexual o que sufren, aun estando cerrados los centros escolares, ciberbullying y miedos ante la posible vuelta; aquellos que están sufriendo problemáticas psicológicas y emocionales debido a las medidas de confinamiento social y que se pueden manifestar en episodios como agorafobias, claustrofobias, trastornos alimenticios o ansiedad. En el apartado de la alimentación, el cierre de las escuelas puede estar desencadenando alteraciones en las dietas de los niños y niñas. El consumo de alimentos altamente calóricos, azucarados, carbonatados y con alto contenido en sal está siendo un claro denominador común en muchos hogares durante estas semanas de aislamiento. Ello, combinado con un cambio en las rutinas de ocio donde el acceso a los parques infantiles e instalaciones deportivas es limitado o inexistente, y el abuso del consumo de pantallas, videojuegos e incluso del propio cambio a la docencia online, probablemente generará problemas de salud importantes en la población infantil a tener en cuenta en corto y medio plazo (Rundle et al., 2020).

En el apartado psicológico, el impacto en niños y adolescentes se manifiesta desde una situación de estrés provocada por esa duración prolongada sin contacto con sus iguales, con elevadas tasas de aburrimiento, información inadecuada, miedo a la enfermedad, al contagio de sus seres queridos y a la pérdida de empleo de sus progenitores (Wang et al., 2020). En un estudio llevado a cabo con población joven en España e Italia (dos de los países más afectados por el Covid-19 en la Unión Europea y donde las medidas de confinamiento han sido más drásticas) se ha detectado cómo los síntomas de aburrimiento, irritabilidad, inquietud y sensación de soledad estuvieron bastante presentes, viéndose acrecentados cuando los padres no conseguían manejar ciertas situaciones; dando como consecuencia episodios emocionalmente problemáticos que afectaron al bienestar psicológico del núcleo familiar (Orgilés et al., 2020).

Hay quienes plantean que las medidas de cierre escolar deberían ser aliviadas en cuanto las autoridades sanitarias lo permitan. Desde aquí, el horizonte debe paliar la desigualdad considerando la combinación de la enseñanza no presencial con la presencial adecuando las medidas de distanciamiento e higiene adecuadas. Los países en las etapas iniciales de las medidas de mitigación tienen la oportunidad de ser líderes, priorizando a los jóvenes y estableciendo estrategias para garantizar de manera proactiva que los niños estén en el centro de las respuestas futuras. Todo ello con el objetivo de lograr un equilibrio y protegiendo a los más expuestos sin sacrificar el futuro de la próxima generación (Armitage y Nellums, 2020).

Mientras llegan esas medidas de desescalada y vuelta a una normalidad parcial donde se permita cierto contacto social y cierta reapertura escolar, el profesorado aparece como 
salvavidas ante la situación de indefensión de la infancia y juventud. Son el punto de unión con la normalidad y sirven de asesoramiento continuo tanto a sus propios alumnos como a los padres y madres en esta difícil tarea. Con el trabajo programado de las escuelas y la colaboración familiar se pueden poner en práctica programas educativos a distancia con materiales diseñados, pautas de higiene y alimentación, ejercicio físico en el hogar y desarrollo de la autosuficiencia y autogestión (Wang et al., 2020). Se abre ante así una oportunidad de alcanzar más rápida y eficazmente la madurez del estudiante.

La principal medida que se ha tomado, como ya hemos ido señalando, ha sido la implementación rápida de un modelo de educación a distancia basada en la utilización de recursos tecnológicos. Ésta conlleva retos que se traducen en una multiplicidad de casuísticas dependiendo del nivel educativo. Los niveles de autonomía, el dominio de habilidades, la competencia digital, el número de estudiantes por grupo o los recursos con los que se cuente, afectarán y dibujarán un sinfín de escenarios y relaciones educativas (Sánchez Mendiola et al., 2020). Sin embargo, es preciso advertir y alertar de que este proceso de digitalización de la docencia puede no estar llevándose a cabo de una manera correcta y satisfactoria en lo que a planteamiento y ejecución didáctica se refiere. Como señalan Zhou y otros (2020) algunos docentes han podido realizar una transposición didáctica del aula física al aula virtual sin realizar las adaptaciones requeridas en cuanto a material, temporalización, carga de trabajo, formas de interacción profesor-alumno correctas. Además, la diversidad de alumnado presente en las aulas y los nuevos formatos de trabajo en entornos virtuales requieren de un alumnado autónomo y con capacidad para el autoaprendizaje que en muchos casos no se ha alcanzado antes en la enseñanza presencial. Si a eso le unimos una baja supervisión parental y escasos recursos telemáticos, el fracaso en el proceso de enseñanza-aprendizaje se puede estar encontrando en un alto porcentaje de alumnos. En muchos casos, el paso a la docencia online se hizo de manera prematura, acelerada, sin la preparación adecuada y sin la reflexión pedagógica necesaria para acometer el paso a los entornos virtuales de enseñanza. La consecuencia de todo esto puede dar lugar a una paradoja. Es lo que se señala en el estudio de Vlachopoulos (2020): instituciones educativas, profesorado y alumnado deben tener muy presente que este grado de digitalización de la enseñanza y con esta rapidez de acción no se ha dado nunca antes. De este repentino cambio aparecerá la oportunidad de probar los límites y alcances de una docencia online pero siempre que se lleven a cabo bajo las condiciones adecuadas, es decir, con recursos y competencias en y para todos los actores de los sistemas educativos. De no ser así, el caos será el protagonista y los resultados que se analicen de esta nueva realidad estarán sesgados por una omisión de la desigualdad. Sin embargo, cuando pase este estado de excepcionalidad, los teóricos de la educación, docentes y comunidad educativa en general, deben tener claro que ni la enseñanza online es el paradigma del progreso ni, en los casos en las que ésta tenga una mala implementación, debe dejar de ser un horizonte por perseguir.

La población universitaria también está padeciendo las consecuencias del Covid-19. La Universidad ha tenido que afrontar un cambio repentino, moviéndose de la presencialidad a la no presencialidad. Esta educación mediada por tecnología ha solicitado de un esfuerzo de aprendizaje exprés para muchos docentes anclados en la enseñanza tradicional, entendiendo por tal la interacción personal en un aula presencial dentro de un contexto formal de enseñanza-aprendizaje en un entorno físico. Desde las instituciones universitarias y dependiendo de los recursos se ha tratado de ofrecer una respuesta lo más eficaz y coordinada posible para que el curso académico se desarrollara con relativa 
normalidad. No obstante, siempre existen pérdidas por el camino y situaciones de estrés docente que deben asumirse paralelas a las situaciones personales vividas en el contexto de la pandemia (Sánchez Mendiola et al., 2020). La pérdida o adaptación de clases, las nuevas rutinas, vivir en entornos rurales o urbanos y convivir con circunstancias familiares donde la enfermedad o las consecuencias económicas acechaban han sido factores que han generado diversos niveles de estrés y problemáticas de salud emocional a los estudiantes. Las perspectivas de un futuro indeterminado son un pensamiento con el que una gran parte de la población estudiantil universitaria ha tenido que convivir en las últimas semanas (Cao et al., 2020).

Finalmente, a todo lo señalado hemos de añadir la natural disparidad de niveles de digitalización en todo el mundo. No todos los sistemas educativos tienen garantizado el mismo nivel de éxito en la modalidad de educación a distancia online puesto que no todos los países poseen la misma estructura. En un informe realizado por el Banco de Desarrollo de América Latina y la Comisión Económica para América Latina y el Caribe dependiente de Naciones Unidas (2020) se establece una evaluación acerca de la digitalización de América Latina y el Caribe para hacer frente a las consecuencias de la crisis provocada por el Covid-19. En dicho informe se detalla la posición que ocupan en el mundo en relación con lo que se denomina ecosistema digital. En primer lugar, estaría América del Norte, seguido de Europa Occidental, en una posición intermedia estarían los Estados Árabes y Europa del Este, a continuación, en la clasificación estarían Asia-Pacífico y América Latina y el Caribe. En último lugar se situaría África. Los problemas de falta de conectividad y habilidades digitales de los responsables de la educación, unido a la falta de plataformas virtuales son los protagonistas del estado de la educación a distancia en América Latina y el Caribe. Como se señala en el informe, países como Argentina se han replanteado la utilización de la televisión y la radio para lograr niveles de democratización de la enseñanza ante la situación. Y es que, el derecho a la educación, con independencia de que adaptaciones se hayan tenido que desarrollar, debe estar salvaguardado. Si la docencia a distancia se ha impuesto en formatos de digitalización, las administraciones educativas deben velar porque todo alumnado tenga acceso a la misma y en las mismas condiciones (Bekerman y Rondanini, 2020; Cotino Hueso, 2020). Lo que queda por resolver es si este reto se está consiguiendo, y si es imperativo de las administraciones educativas atender la diversidad de la población estudiantil. En nuestra opinión se trata de una necesidad inalienable del Estado. Además, es preciso que las administraciones combatan la brecha digital y la brecha social como objetivos prioritarios en materia de inversión educativa.

Lamentablemente, en esta situación de incertidumbre, no son pocos los elementos que quedan sin concretar y que con el pasar de los meses irán generando diferentes disyuntivas. Esperemos que las decisiones que se vayan tomando ofrezcan una respuesta ante la curva de desigualdad educativa que puede dejar al descubierto el Covid-19.

\section{Método}

El marco internacional que ofrece este estudio tiene como meta lograr mayor cota de comprensión sobre las implicaciones que está generando para el ámbito educativo la pandemia provocada por el Covid-19. Dada la novedad del objeto de estudio, la inmediatez de los datos, y lo rápido que evoluciona, se ha optado por utilizar una metodología de corte cualitativo y exploratorio, en 23 países y 4 continentes. La investigación mide la 
percepción de informantes clave de cada país acerca de las consecuencias que el cierre de las escuelas ha generado.

La utilización de informantes clave en la investigación cualitativa es una posibilidad para un acercamiento certero a la realidad social estudiada (Ander-Egg, 1995). Por sus cualidades, los informantes clave realizan una función de apadrinamiento hacia el investigador, por el número y calidad de las vivencias y datos que estos pueden transmitirle en beneficio de su estudio (Monistrol, 2007; Robledo y Martín, 2009). Desde un correcto proceder metodológico, los informantes deben ser considerados como auténticos individuos con capacidad reflexiva y benefactores de datos (Sánchez Silva, 2005). No serán el objeto de investigación sino el medio por el cual llegar al conocimiento.

En cuanto a los criterios de selección de los informantes clave, estos estuvieron determinados por la relación con el objeto de estudio y su accesibilidad, de acuerdo con un procedimiento de muestreo propositivo (Alejo y Osorio, 2016). A continuación, se presenta la enumeración de criterios de selección de la muestra participante:

- Poseer una formación académica y profesional acreditada y acorde con el objeto de estudio.

- Dominio y actualidad de conocimientos sobre la realidad analizada.

- Aproximación geográfica o contacto directo con la localización objeto de estudio.

- Mantener relación profesional y/o de investigación con actores del sistema educativo.

- Voluntariedad y compromiso explícito de participación como informante.

- Conocimiento de la técnica de recogida de datos para el estudio.

- Accesibilidad y disponibilidad online ${ }^{4}$.

Como señala Mendieta Izquierdo (2015), los criterios de selección de la muestra son determinantes para el correcto proceder en la investigación cualitativa y para el rigor en el análisis de la realidad a estudiar. Así, para este estudio, la muestra de informantes clave se compuso de 23 sujetos, de los cuales 14 fueron mujeres y 9 fueron hombres. La media de edad se situó en los 49,5 años. La selección de esta muestra permitió obtener una percepción de la nueva realidad educativa de diferentes países repartidos por cuatro continentes: Ecuador, Chile, Colombia, Uruguay, Cuba, República Dominicana, Paraguay, Brasil, El Salvador, Perú, Guatemala, México, Marruecos, Senegal, Túnez, Noruega, España, Portugal, Suecia, Italia, Francia, Australia y EEUU ${ }^{5}$. Todos los informantes participaron de forma voluntaria tras ser informados de los objetivos del trabajo, sin recibir incentivos materiales por su colaboración y con disponibilidad online plena. La naturaleza y perfil de la muestra participante en el estudio estuvo distribuida de la siguiente forma: 5 maestros de Educación Primaria, 5 profesores de Educación Secundaria, 6 profesores de Universidad, 4 psicólogos educativos y 3 sociólogos. Además de su

\footnotetext{
${ }^{4}$ Dadas las inusuales circunstancias de excepcionalidad basadas en el aislamiento y la limitación de movilidad de las personas en la mayoría de las regiones del mundo, -durante la realización de este trabajo de investigación- la modalidad online a distancia se impone en todas las actividades. Ello también afecta al proceso investigador de este trabajo y por ende a la relación y flujos de comunicación entre el grupo de investigadores responsables de este trabajo y los sujetos que conforman la muestra participante.

${ }^{5}$ En el caso de Estados Unidos, la localización geográfica de los datos y el informante clave se corresponden únicamente con el Estado de Washington.
} 
titulación académica, todos poseen formación específica en Derechos Humanos y Desarrollo Social. Los informantes, según el país, presentan los siguientes perfiles: Ecuador: socióloga e investigadora social independiente con 40 años de experiencia. Exvicepresidenta del Comité de Derechos del Niño de Naciones Unidas; Chile: docente e investigadora en la Universidad Autónoma de Chile con 12 años de experiencia y participante en numerosos estudios sobre educación.; Colombia: docente e investigadora en la Universidad San Buenaventura de Bogotá con 25 años de experiencia; Uruguay: docente en centro público de zona desfavorecida con 20 años de experiencia, colaboradora de proyectos educativos internacionales.; Cuba: psicóloga docente e investigadora en la Universidad de La Habana con 15 años de experiencia y autora de múltiples publicaciones.; República Dominicana: directora de un centro educativo privado con 17 años de experiencia; Paraguay: docente e investigadora en Universidad del Este con 30 años de experiencia. Autora de numerosos estudios, conferencias y publicaciones; Brasil: profesora de un centro de educación primaria en zona desfavorecida con 15 años de experiencia. Colaboradora en proyectos sociales; El Salvador: profesor de educación secundaria en un centro público con 32 años de experiencia; Perú: sociólogo y director de proyectos sociales con población infantil con 25 años de experiencia; Guatemala: sociólogo y presidente de organización social de infancia con 30 años de experiencia; México: docente e investigadora en Universidad Nacional Autónoma de México con 15 años de experiencia. Autora de investigaciones y publicaciones sobre infancia en riesgo; Marruecos: profesor de educación secundaria, presidente de organización social de infancia, gestor de proyectos con adolescentes en contextos de riego con 25 años de experiencia; Senegal: profesor de educación primaria, presidente de organización social de infancia y gestor de proyectos con niños en contextos de riesgo con 15 años de experiencia; Túnez: profesora de educación secundaria y coordinadora de proyectos educativos internacionales con 25 años de experiencia; Noruega: psicólogo en centro de protección de adolescentes en riesgo con más de 30 años de experiencia; España: docente e investigador en la Universidad, coordinador de proyectos educativos internacionales con 40 años de experiencia. Autor de diversas publicaciones, estudios y conferencias; Portugal: profesora de educación secundaria, presidenta de organización social de infancia en contextos de riesgo con 15 años de experiencia; Suecia: psicóloga con 50 años de experiencia, activista; Italia: psicólogo y director en centro de protección de adolescentes en riesgo con 30 años de experiencia; Francia: profesora de educación primaria con 5 años de experiencia. Investigadora internacional; Australia: docente e investigadora en la Universidad de Sídney con 25 años de experiencia. Participante en proyectos de investigación diversos, conferenciante y autora de múltiples publicaciones; EE.UU.: Full professor en la Universidad, director de programas internacionales, con más de 30 años de experiencia docente e investigadora.

En lo que concierne a la recogida de datos, se realizó mediante un cuestionario de preguntas abiertas y cerradas distribuido de manera online y disponible en 5 idiomas (español, inglés, francés, portugués e italiano). El cuestionario fue diseñado de acuerdo con una secuencia lógica de 31 preguntas acordadas por el equipo investigador que dieran respuesta a las siguientes categorías de análisis, necesarias para dar coherencia a la matriz del estudio (Cerrón, 2019):

- Tiempos en la ejecución, anticipación o demora de medidas.

- Carácter democrático-participativo de las medidas educativas tomadas. 
- Adaptación curricular, didáctica y metodológica.

- Infraestructura y recursos para una adecuada formación a distancia.

- Percepciones acerca del profesorado.

- Percepciones sobre el entorno familiar.

- Propuestas alternativas.

Se eligió la utilización de cuestionarios como instrumento de investigación al considerarlos una eficiente técnica de recogida de datos en la investigación educativa, al generar un procedimiento estandarizado que sirve para recoger y analizar los datos de una manera que permite describir, explorar, y explicar una serie de características (Casas Anguita et al., 2003).

Tras la recepción de los datos, se llevó a cabo un análisis de contenido (Piñuel Raigada, 2002) contemplando las ideas y llevando a cabo un procedimiento controlado de inferencia transitando de una fase descriptiva a otra interpretativa propia del método. En el apartado de resultados se muestran las percepciones más representativas de los informantes clave de acuerdo con las categorías construidas, cumpliendo así con el objetivo de este trabajo: ofrecer una perspectiva internacional sobre el proceso de cierre de escuelas por el Covid19 y sus posibles consecuencias educativo-sociales a través de las percepciones de informantes clave.

\section{Resultados}

\subsection{Tiempos en la ejecución, anticipación o demora de las medidas}

Los resultados adscritos a esta categoría pretenden presentar las percepciones de los informantes clave en relación con los tiempos que cada país ha ido anticipando o demorando el cierre de escuelas, el inicio y duración de las medidas de cierre y la reconfiguración del calendario escolar.

En este sentido, el 70\% de los informantes considera que en su país el cierre de las escuelas no se ha demorado mientras que el 30\% (Noruega, Italia, Francia, México, Brasil, Guatemala) considera que sí. A ello se ha de añadir que Australia y Suecia no cerraron sus escuelas. En Australia cerraron solo 2 escuelas públicas y muchas escuelas privadas que no estaban obligadas; sin embargo, de manera generalizada muchos estudiantes optaron por quedarse en casa. En el caso de Suecia solo cerró la Universidad. Para las demás localizaciones, los informantes aportan que el cierre generalizado se produjo durante la primera quincena de marzo. Por otro lado, en cuanto a la duración del cierre, los informantes señalan en todos los casos que estará supeditado a la evolución de la pandemia. En unos países se está optando por el cierre indefinido, en otros casos por plazos prorrogables, otros plantean una desescalada por zonas sin contagio. Finalmente, ante una posible reconfiguración del calendario escolar, el $74 \%$ de los informantes plantean que sí se llevará a cabo, mientras que un $26 \%$ señala que no. Las opciones que se barajan de cara a una nueva configuración tienen que ver con: prolongar el calendario escolar hasta el máximo posible (Francia o Túnez), el adelanto o suspensión de vacaciones (Chile o Guatemala), evaluar sobre el primer y segundo trimestre (Marruecos), aplazar la prueba de acceso a la universidad (Portugal), aplazar la presencialidad a septiembre (Uruguay, Paraguay o Perú) y preparar una metodología híbrida para los meses de octubrenoviembre (Estados Unidos). También hay países en los que hasta la fecha no se percibe 
que haya nada previsto (Colombia, Senegal, Cuba, República Dominicana, El Salvador, Australia o Suecia).

\subsection{Carácter democrático-participativo de las medidas educativas tomadas}

Los resultados adscritos a esta categoría hacen mención a las percepciones de los informantes clave sobre qué organismos o autoridades competentes han participado en la decisión del cierre de las escuelas, así como el grado de consenso alcanzado con los múltiples actores que componen el contexto educativo de cada país y la invitación o no a participar en la toma de decisiones respecto a estas medidas.

El 63\% de los informantes respondieron que la decisión de cerrar los centros educativos se tomó desde el gobierno nacional o federal en sintonía con lo acordado por los ministerios o consejos competentes en materia de sanidad. En cambio, el $22 \%$ señaló que esta decisión fue tomada por las alcaldías o municipalidades y el $9 \%$ por el gobierno de los estados (Brasil y Washington, EE.UU.). Respecto al grado de participación de los actores que componen el contexto educativo en la toma de decisiones sobre el cierre de las escuelas, el $60 \%$ de los informantes considera que en su país sí se ha invitado a participar a responsables y personas directamente relacionadas con el ámbito educativo. El 40\% considera que no ha sido así. En los casos en los que sí se ha producido ese acercamiento, los informantes clave consideran que en una escala del 1 al 5 el grado de consenso estaría situado en 3,16 puntos. Cabe mencionar, en el caso de Brasil, que el informante señala que ciertas escuelas se adelantaron al cierre por vía de los gobiernos locales sin coordinación con el gobierno central. El posicionamiento y discurso del Gobierno Federal de Brasil se basó desde el inicio en priorizar los intereses de la economía, oponiéndose a una paralización de muchos sectores como el escolar por vía del confinamiento. A diferencia de otros países, no hubo acción colectiva y los gobiernos estatales y municipales tomaron decisiones propias basadas en alertas y datos proporcionados por agencias o profesionales vinculados al área de la Salud. Lo visto hasta ahora evidencia un escenario de discordancia política más que de autonomía.

\subsection{Adaptación curricular, didáctica y metodológica}

Los resultados que se relacionan con esta categoría muestran las percepciones de los informantes clave sobre cómo ha sido la adaptación de objetivos y contenidos curriculares, cómo ha sido la adaptación didáctica y qué medidas se han tomado con respecto a la evaluación y calificación de los estudiantes en las diversas etapas educativas. Por otro lado, también se señala la percepción acerca del lugar del alumnado con necesidades educativas especiales en el diseño de estas medidas.

El $70 \%$ de los informantes señala que en sus países sí se ha llevado a cabo cierta modificación o adaptación didáctica. Esas modificaciones se basan sobre todo en una docencia online. En este caso, en países como Noruega, Francia, Italia, EE.UU., Uruguay y Paraguay hay una apuesta decidida, según los informantes, por la elaboración de contenidos digitales. En un porcentaje más bajo de los casos se encuentran las adaptaciones hacia una enseñanza por televisión y la priorización de unos contenidos sobre otros (materias instrumentales básicas). Senegal, Cuba, Túnez, México y Guatemala son ejemplos donde los informantes señalan que se ha hecho bastante énfasis en la preparación de materiales para ser retransmitidos por televisión para llegar a más hogares. Por otro lado, destacan iniciativas como las de Ecuador donde se ha establecido un currículo de emergencia con objetivos de aprendizaje priorizados e incorporación de actividades de 
contención emocional. Ello está circunscrito al denominado Plan Aprendamos en Casa donde antes de la aplicación del currículo se desarrollan actividades de apoyo emocional y refuerzo de los aprendizajes previos. Así mismo, en países como Chile o Brasil existe una gran diversidad de casos y diferencias entre la capital del país y el resto de zonas. El acceso a una enseñanza privada o pública que está determinada por una población estudiantil con diferentes recursos y un profesorado con diferencias en los materiales para su docencia determina las adaptaciones que se están llevando a cabo.

En lo concerniente a la evaluación y promoción del alumnado, existe diversidad de opiniones. Hay cierta representatividad en torno a evaluar a través de las tareas que se están enviando desde diferentes medios, síncronos y asíncronos, para que los estudiantes realicen en sus hogares. No obstante, existe una percepción generalizada ofrecida por los informantes de la que se deriva que no existen instrucciones oficiales, claras y homogéneas respecto a la evaluación y mucho menos sobre la calificación y promoción de los estudiantes. Primará la adaptación a la realidad, al contexto, buscándose sobre todo el refuerzo de los aprendizajes previos y que en la medida de lo posible se consiga un nivel aceptable de nuevos contenidos. Además, desde países como Brasil, se plantea la dificultad de evaluar a estudiantes sin el acceso a los materiales educativos y por ende al aprendizaje en igualdad de condiciones. En el caso de España, las autoridades han señalado cierto nivel de laxitud con respecto a la medición de los aprendizajes y a la promoción de los estudiantes, instando a los docentes a aplicar mecanismos empáticos y a adoptar posturas de sintonía con los estudiantes para conseguir un mayor grado de comprensión por la situación.

Finalmente, poniendo el foco sobre el alumnado más vulnerable y con necesidades educativas especiales, en mayor medida, la muestra de informantes consultados declara que no se ha planeado nada adicional por el estado de pandemia. Un grupo menor de informantes percibe que se está actuando en las mismas formas que con el resto del alumnado. Sin embargo, otros informantes describen que el apoyo de las familias como vía compensadora, la realización de adaptaciones individuales y las acciones desempeñadas de los profesionales de educación especial están haciendo salvar en cierta medida la situación. Estos últimos informantes se localizan en los países de: Uruguay, Noruega, Portugal, Ecuador, Francia y Perú.

\subsection{Infraestructura y recursos para una adecuada formación a distancia}

Los resultados concernientes a esta categoría presentan las percepciones de los informantes clave acerca de la presencia/ausencia de medidas sociales y económicas tomadas por las administraciones y los centros educativos para salvaguardar los principios de igualdad y equidad de todos los estudiantes, así como describir las consecuencias que la ausencia de estas medidas puede estar generando en la población estudiantil más vulnerable.

De acuerdo con ello, el 70\% de los informantes considera que, ya sean las administraciones o bien los centros, sí están tomando medidas sociales y/o económicas. Sin embargo, un $30 \%$ tiene una percepción de que ello no es así. Los informantes clave que consideran esto hacen referencia a la situación vivida en Chile, Colombia, Marruecos, España, Brasil y Guatemala. Respecto a las consecuencias que está generando en la población estudiantil más vulnerable, la posible ausencia de medidas sociales y económicas, el $45 \%$ de los informantes señala que la falta de ayudas se deriva en un nulo o insuficiente acceso a internet y otros medios tecnológicos. Desde Brasil se argumenta como contradictorio el 
hecho de que se derive de manera íntegra a un formato de educación basado en TIC, sin que los propios centros hayan tenido en el pasado una dotación como la que ahora se pretende que tengan en todos los hogares del país. Por otro lado, esa ausencia de ayudas también está generando desprotección a nivel físico, de alimentación e higiene, cuestión recalcada por el $18 \%$ de la muestra. Y, por último, se señala la notable bajada del rendimiento académico en la población estudiantil más vulnerable y los problemas de salud mental que pueden llegar a estar padeciendo por la situación de confinamiento e incertidumbre. En el caso de Senegal, se percibe el hecho de que las consecuencias pueden ser el abandono de los estudios debido a las condiciones de pobreza que vive la mitad de la población. Paradójicamente, este aspecto tendría cierto atisbo de coincidencia con lo señalado por el informante clave de EE.UU. Desde su percepción, existe la posibilidad de que ciertos alumnos sin recursos tuvieran que abandonar los estudios universitarios para retomarlos en un futuro.

\subsection{Percepciones acerca del profesorado}

Los resultados adscritos a esta categoría muestran la percepción de los informantes clave de cada país acerca de las facilidades o dificultades que el profesorado está presentando para adaptarse a esta nueva situación, así como las presiones sufridas o lagunas formativas encontradas fruto de esta crisis.

Los resultados de la muestra participante evidencian una división acerca de la adaptación del profesorado a esta mueva situación. El 48\% de los informantes (Colombia, Uruguay, Marruecos, Senegal, Noruega, Suecia, Paraguay, República Dominicana, Ecuador, Estados Unidos y Perú) percibe que el profesorado ha tenido más facilidades mientras que el $52 \%$ percibe que ha tenido más dificultades (Chile, España, Portugal, Italia, Cuba, Túnez, Francia, México, Brasil, El Salvador, Australia y Guatemala). Consideran los informantes que entre las facilidades que se han podido ver representadas están las de una mayor flexibilización del currículum y la entrega en las actividades y tareas de los estudiantes; un mayor grado de contacto y relación educativa gracias a las nuevas tecnologías (Internet) y la posibilidad de brindar apoyo al alumnado. Entre las dificultades, la que mayor peso representa en el cúmulo de percepciones de los informantes es el desconocimiento de las herramientas tecnológicas de los docentes. Esta dificultad además aparece como la más citada en la percepción de las lagunas formativas de los informantes. A partir de ahí se enumeran otras dificultades o limitaciones del día a día que tienen que ver con problemas familiares, pérdida de intimidad, problemas emocionales, problemas económicos por el retraso de su retribución, anuncio de recortes y falta de contacto con algunos estudiantes.

Por último, en el apartado de las presiones que perciben los informantes que puede estar sufriendo el profesorado, la mayoría se derivan de que las familias no comprenden los contenidos y sienten que ellos tienen que ocuparse de la enseñanza. Así mismo, existe cierto grado de presión derivado de la exigencia de que el aprendizaje a distancia siga el ritmo de la enseñanza en el aula presencial, lo que tiene que ver con un sentimiento generalizado de que el trabajo de los profesores se ha multiplicado. A ello debe sumarse la incertidumbre de no saber qué ocurrirá con el curso escolar.

\subsection{Percepciones sobre el entorno familiar}

En lo relativo a esta categoría, los resultados evidencian las percepciones de los informantes clave acerca de la respuesta ofrecida por el entorno familiar ante el cierre de 
las escuelas. Posibilidades de albergar un entorno adecuado de aprendizaje para sus hijos/as, flujos de comunicación con el profesorado y lagunas formativas encontradas fruto de esta crisis. Esta categoría hace mención también a cómo se está afrontando la respuesta al alumnado con necesidades educativas especiales.

En este sentido, la percepción de los informantes es bastante dispersa y solo el $48 \%$ de ellos considera que ha habido una relativa buena respuesta ante la nueva situación educativa. La percepción es que existe una mezcla entre conformidad y resignación por lo ocurrido y de que no existe otra alternativa. Hay cierta preocupación, sobre todo en los casos en los que la familia ha tenido que reorganizarse para ocuparse de los hijos, en los que la cuestión laboral y económica ha afectado de lleno a la conciliación y los casos en los que cierta parte de la población de riesgo (abuelos y abuelas) pasan a ocupar espacios de convivencia con los niños. Este aspecto se destaca desde Italia. Por otro lado, desde Guatemala se hace mención a la vulnerabilidad de las madres que conforman el único elemento parental del grupo familiar y los casos donde ha aumentado los niveles de tensión y maltrato intrafamiliar.

En cuanto a los flujos de comunicación que se mantienen entre familia y profesorado, las llamadas de teléfono, el correo electrónico, aplicaciones de comunicación instantánea como WhatsApp, de videollamadas como Zoom y redes sociales varias son consideradas como los mecanismos más utilizados. Respecto a las lagunas formativas que pueden percibir en las familias para sobrellevar esta nueva situación, los informantes señalan por encima de todo tres: el bajo nivel de formación cultural y académica, lo que implica unos niveles de apoyo y seguimiento en las tareas escolares de los hijos más precario; el desconocimiento en el campo de lo tecnológico; y el desgaste emocional, asociado a una capacidad baja para manejar las situaciones de estrés, aburrimiento, falta de alternativas de ocio y de comprensión de lo ocurrido en el propio seno familiar.

Finalmente, en lo que tiene que ver con la respuesta y alternativas que tiene la familia para desenvolverse en este contexto con hijos/as con necesidades educativas especiales, la respuesta de los informantes se muestra diversa. Los que pueden ofrecer una percepción sólida señalan por un lado el papel de refuerzo y apoyo que asumen las propias familias para sus hijos (Uruguay, Ecuador), por otro lado, las muchas dificultades que se les plantea con este nuevo presente por la falta de ayudas de la administración (Marruecos, Senegal, Italia, México, Guatemala) este aspecto, sin duda, está directamente ligado al desarrollo y evolución de la pandemia y la posibilidad de mantener abiertos o cerrados ciertos servicios de educación especial. Y, por último, los que el sistema brinda cierto refuerzo que compensa las posibles barreras derivadas de la situación (Noruega, Cuba).

\subsection{Propuestas alternativas}

Los resultados de esta categoría recogen aquellas medidas e iniciativas de corte alternativo, diferentes a las actuales, propuestas por los informantes en su condición de conocedores del contexto, que son susceptibles de ser implementadas para la mejora de la situación actual, haciendo énfasis en las situaciones de mayor vulnerabilidad social y educativa. Puede verse un resumen de estas propuestas en la figura 1. 


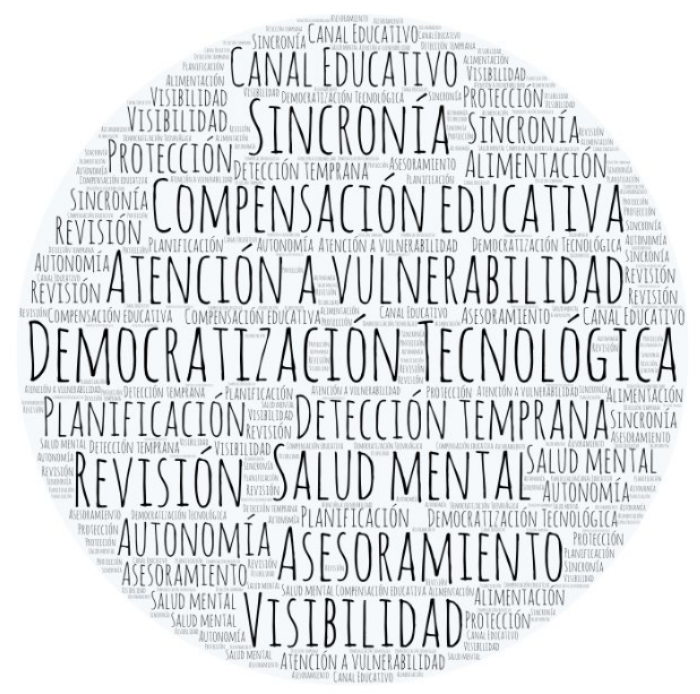

Figura 1. Análisis de contenido. Propuestas alternativas Fuente: Elaboración propia.

Los informantes argumentan como necesario alcanzar un mayor grado de democratización tecnológica. Ello pasa por facilitar el acceso a internet y la tenencia de dispositivos de interacción online para todos y para todas. También se plantea la posibilidad de un incremento de la sincronía en las modalidades de conexión online, logrando así mayor cota de parecido con la clase habitual presencial. Por otro lado, plantean la emisión masiva de contenido educativo curricular a través de la creación específica de canales en distintos medios de comunicación. Además, se aboga por una reestructuración del calendario escolar para que el nuevo inicio de curso cuente con un mes previo de preparación a una nueva modalidad, pensada y reflexionada, que se adapte mejor a las circunstancias. Esa nueva realidad pasaría por un aumento en el diseño de actividades auto-correctivas que fomentaran la autonomía del alumnado y las familias. Señalan la necesidad de reorientar los fines educativos hacia la salud mental, sobre todo en los casos de alumnos con mayor vulnerabilidad. Por otro lado, señala que se deben plantear formas de asesoramiento familiar, generar procesos de atención domiciliaria para el alumnado más vulnerable, complementado con programas de refuerzo de la alimentación infantil, y protección contra todos los tipos de violencia que pueda sufrir el niño o adolescente. Todo con el objetivo de aumentar la visibilidad de los más vulnerables. Finalmente, con una mirada a la vuelta a la normalidad, los informantes plantean el diseño de nuevos programas de detección precoz y de compensación educativa.

\section{Discusión y conclusiones}

El objetivo de este trabajo enunciado ya en apartados anteriores era, a través de una investigación exploratoria, ofrecer una perspectiva internacional sobre el proceso de cierre de escuelas por el Covid-19 y sus posibles consecuencias educativo-sociales a través de las percepciones de informantes clave. De acuerdo con ello se ha establecido un estudio exploratorio, descriptivo y de corte cualitativo en el que han participado informantes clave de 23 países. A través de los datos recogidos mediante un cuestionario online, se han presentado los resultados conforme a siete categorías que ofrecieran estabilidad y coherencia al estudio. 
Cabe destacar que, según las percepciones de los informantes clave consultados, la salud de los principios de igualdad de oportunidades y equidad educativa se está viendo perjudicada. Las posibilidades de mejora y ascenso social que provee la educación en un sistema que apuesta por estos principios se ven hipotecadas en estos momentos. Las consecuencias que trae consigo la pandemia provocada por el Covid-19 y que está forzando a las autoridades competentes al cierre de las escuelas se está llevando las posibilidades de acceder a niveles óptimos de desarrollo educativo a muchos alumnos y alumnas de todo el mundo. Sin embargo, a pesar del cierre de las escuelas, una de las consecuencias sociales que podemos sacar de esta situación es el escepticismo socioeducativo, en tanto que consideramos que, a pesar de vivir una excepcionalidad social, los sistemas educativos siguen luchando por mantener una falsa normalidad, cuando es evidente que nada sigue siendo normal. Si antes de la pandemia había desigualdad social, contra la que con mejores o peores resultados la escuela luchaba a través de programas de refuerzo, seguimiento, becas, compensación, ayudas, maestros comprometidos presencialmente, etc., ahora, eso se está perdiendo, agravando todavía más la brecha social y la injusticia.

En este sentido, familias e infancia vulnerable se encuentran en primera línea de una playa cuya ola de pobreza y falta de futuro, en muchos casos ya se los ha llevado por delante. La inversión y ayudas también se deben dar a tiempo real en el sector educativo público, ayudando a apuntalar una bisagra social situada entre lo puramente sanitario y lo económico de un país. El sistema educativo público debe abanderar la lucha por la equidad y los derechos sociales. El sistema educativo público, al igual que los servicios sociales o la sanidad, debe ser garante de los principios democráticos de igualdad y justicia social, y permanecer, si cabe, más atento a los intentos de menoscabo o precarización, que ya se vienen anunciando en forma de hipotéticos recortes que, desde una economía neoliberal golpeará con fuerza a los sectores más débiles de la sociedad.

Sólo así, con la apuesta seria por la educación pública, con el aumento de inversión educativa y con el compromiso de apoyar la escuela y a los parias que han condenado a vivir en la parte baja de la brecha educativa y en el lado oculto del sistema escolar, se garantizará una calidad educativa por medio de la igualdad de oportunidades y con ello un mantenimiento de la equidad social (Pascual Bario, 2006). Los estándares de disponibilidad, accesibilidad, aceptabilidad y adaptabilidad en educación (Lara Caballero, 2017) deben asegurarse hoy más que nunca para garantizar el derecho a la educación. Así, esa mirada debe trazarse de manera desigual, no tratando igual a los desiguales (De la Cruz Flores, 2017), ayudando a quien más lo necesite y forzando al sistema para no dejar atrás a los más débiles de la cadena.

El cierre de las escuelas y la reconfiguración de los calendarios escolares con las medidas concretas que lo acompañan generan una gran diversidad, cierta incertidumbre y adaptación continua en función del desarrollo de la enfermedad y de las medidas y urgencias económicas presentes en cada país. Este es uno de los grandes peligros detectados en este estudio. Si no se trata con el cuidado y la importancia debida, la crisis sanitaria y económica provocada por el Covid-19 generará una gran desigualdad educativa que tardará años en compensarse. Esta desigualdad educativa, en nuestra opinión, desencadenará una desigualdad social, al bloquear la ascensión social y restringir la igualdad de oportunidades eliminado el acceso a los estudios superiores de un gran número de jóvenes de clases desfavorecidas privándoles de la única palanca social para mejorar la vida cuya responsabilidad es del sistema público. La presencia de los actores educativos en la toma de decisiones se concibe como existente en cierta medida, aunque la 
multiplicidad de formatos de gobierno, cogobierno y autonomía derive un escenario de difícil coordinación con las consecuencias de estrés para el sistema educativo en general que ello desencadena.

La adaptación metodológica está más que presente en los países en los que se ha decretado el cierre de escuelas. En estos casos la modalidad a distancia online apoyada en el uso de diferentes recursos tecnológicos con conexión a internet es la más utilizada, complementada en algunos casos con el uso de medios de comunicación de masas como la televisión. En el caso de la primera, requeriría más de un debate epistemológico a causa de la práctica docente que se está llevando actualmente con transposiciones didácticas de la clase presencial a la virtual que en nada tiene que ver con lo que sería una verdadera formación online ${ }^{6}$. Añadido a esto, se advierten diferentes grados de dificultad entre los informantes clave respecto a una problemática común: aquella que tiene que ver con el colectivo más vulnerable sin el acceso adecuado a los recursos digitales. Ello obliga tanto a las familias como a los docentes a generar alternativas o a decaer en el intento ante situaciones de elevada desigualdad intraterritorial. En el caso de los docentes, este nuevo contexto educativo, sin duda, es una prueba para su identidad profesional y su profesionalidad docente. Si Bolívar y otros (2014) ya señalaban la necesaria reconfiguración de esta identidad basada en el aprendizaje de nuevas competencias en la sociedad de la información; ahora el reto es si cabe más determinante. Como señalan Villafuerte y otros (2020), ante la situación de excepcionalidad educativa provocada por el Covid-19 el docente debe ser soporte, promotor de la resiliencia, guía en lo académico, animador, asesor emocional y garante de la organización y coherencia institucionales.

Si en una situación ordinaria de enseñanza, la coordinación, apoyo y trabajo en la misma dirección del profesorado y familias es necesaria; en un escenario como este se hace más que urgente para la detección de necesidades en el alumnado. Por ello, los flujos de comunicación que se están estableciendo de manera habitual, según muestran los resultados (redes sociales, teléfono, correo, aplicaciones telemáticas) deben reforzarse para aquellos hogares que no dispongan de ellas. Por otro lado, debe darse un especial seguimiento a los casos en los que la vulnerabilidad entronca con situaciones en el seno de la familia (Díaz López y Pinto Loría, 2017). La institución escolar, bajo las nuevas condiciones, tiene el reto de no fallar y buscar los cauces necesarios para lograr paliar la situación de indefensión que pueden estar viviendo muchos jóvenes y niños.

El horizonte que podemos observar hoy aparece difuminado, nada claro. Existe una incertidumbre marcada por un caso sin igual y que tiene en jaque a todos los elementos de un sistema interconectado, globalizado. Hoy empieza a hacerse visible una realidad, aquella que tiene que ver con un principio de desigualdad que puede verse incrementada a pasos agigantados marcando el destino de muchos niños y jóvenes. La paradoja educativa nos dice que en ocasiones la educación puede ser generadora de desigualdad, pero que a la vez es la mejor herramienta para reducirla y el gran motor del cambio social (Murillo y Hernández, 2014).

La punta del iceberg de la desigualdad parece estar mostrándonos un excesivo énfasis por una transformación de la cultura escolar en una cultura escolar digital en la que ni todos

${ }^{6}$ Timeline (Chile) Especial. "Cristian Celdón, experto en Educación: "Una clase por zoom no es una formación online". Recuperado de: https://timeline.cl/2020/04/cristian-celedon-experto-en-educacion-una-clase-porzoom-no-es-una-formacion-online/ Fecha de consulta [06/05/2020]. 
ni todas las partes cuentan con las mismas posibilidades (Beltrán y Venegas et al., 2020). Posibilidades que, en definitiva, dejan a muchos actores sin voz, sin capacidad de respuesta, sin posibilidades de construir la parte del mundo que les correspondería por derecho. La hiperconexión digital, la exigencia de evaluar contenidos y no competencias, el cumplimiento de tiempos y calendario escolar es una realidad hoy, a pesar de las dificultades. Sin embargo, se precisa darle forma a una escuela siempre informe, en construcción constante, donde se planteen los principios democráticos universales, la ética ciudadana y colectiva, donde los más necesitados encuentren los puentes necesarios para salir de la indignidad social. La escuela no es solo el lugar donde se transmiten contenidos (que algunos, con una exagerada tecnofilia reducen a lo online) sino donde se construyen identidades alrededor del maestro con palabras y pensamientos participados; es un aquí y un ahora, un tiempo, pero también un espacio compartido donde todos tiene que tener presencia y participación. En relación a la participación, compartimos la idea de Simón y otros (2019) al considerar que la participación brinda igualdad de oportunidades, no solo de aprendizaje, sino también de implicarse en situaciones sociales, de vivir experiencias y relaciones positivas en las aulas y en los centros, constituyéndose la escuela como un lugar del buen trato. Esta es la tarea de reconstrucción de la escuela en tiempos del Covid-19.

Si no somos conscientes de estas debilidades; si como docentes y como sociedad no somos capaces de mirar a los ojos a la adversidad y denunciar la situación que miles o quizás millones de niños y niñas sufren en cuanto al acceso a la educación, es que estamos fracasando como sociedad y como especie humana. Hoy, en los tiempos del Covid-19, hace falta más que nunca otros héroes, aquel profesorado crítico, comprometido, intelectual transformador (Giroux, 1988), que desde su práctica educativa se transforme en un activista social. Las prácticas son las que validan las teorías. Por las acciones e intenciones se conocen a los profesores. Los informantes clave han marcado el camino, cercanos en algunas cuestiones, y diferentes en otras. Pero se vislumbra un punto en común: la rotunda seguridad en que, si la educación no es para el ser humano, si la educación no es para el cambio social, si la educación no es para la justicia social, no es educación, y el trabajo será en vano.

\section{Referencias}

Alejo, M. y Osorio, B. (2016). El informante como persona clave en la investigación cualitativa. Gaceta de pedagogía, 35, 74-85.

Álvarez Zarzuelo, M. A. (2020). El confinamiento de niñas y niños en España en 2020 por la crisis del Covid-19: Propuestas desde la educación social escolar para la vuelta al centro escolar. RES: Revista de Educación Social, 30, 457-461.

Ander-Egg, E. (1995). Técnicas de investigación social. Editorial Lumen

Armitage, R. y Nellums, L. B. (2020). Considering inequalities in the school closure response to Covid-19. The Lancet Global Health, 8(5), e644.

Arriazu, R. y Solari, M. (2015). El papel de la educación en la estrategia Europa 2020: Una aproximación crítica. Revista de la Asociación de Sociología de la Educación-RASE, 9(1), 149161.

Banco de Desarrollo de América Latina y Comisión Económica para América Latina y el Caribe. (2020). Las oportunidades de la digitalización en América Latina frente al Covid-19. Naciones Unidas. 
Bekerman, U. y Rondanini, A. (2020). El acceso a internet como garantía del derecho a la educación. Diario DPI Suplemento Salud, 58, 1-7.

Beltrán, J., Venegas, M., Villar-Aguilés, A., Andrés-Cabello, S., Jareño-Ruiz, D. y de GraciaSoriano, P. (2020). Educar en época de confinamiento: La tarea de renovar un mundo común. Revista de Sociología de la Educación, 13(2), 92-104. https://doi.org/10.7203/RASE.13.2.17187

Berger, Z. D., Evans, N. G., Phelan, A. L. y Silverman, R. D. (2020). Covid-19: control measures must be equitable and inclusive. BMJ, 368, 1-2. https://doi.org/10.1136/bmj.m1141

Bolívar, A., Domingo Segovia, J. y Pérez-García, P. (2014). Crisis and reconstruction of teachers' professional identity: The case of secondary school teachers in Spain. The Open Sports Sciences Journal, 7, 106-112.

Cao, W., Fang, Z., Hou, G., Han, M., Xu, X., Dong, J. y Zheng, J. (2020). The psychological impact of the Covid-19 epidemic on college students in China. Psychiatry Research, 287(112934), 15 .

Casas Anguita, J., Repullo Labrador, J. y Donado Campos, J. (2003). La encuesta como técnica de investigación. Elaboración de cuestionarios y tratamiento estadístico de los datos. Atención Primaria, $31(8)$, 527-538.

CEPAL. (2020). América Latina y el Caribe ante la pandemia del Covid-19: Efectos económicos y sociales. CEPAL.

Cerrón, W. (2019). La investigación cualitativa en educación. Horizonte de la Ciencia, 9(17), 1-8.

Cervantes Holguín, E. y Gutiérrez Sandoval, P. R. (2020). Resistir la Covid-19. Intersecciones en la educación de Ciudad Juárez, México. Revista Internacional de Educación para la Justicia Social, 9(3), 7-23.

Cotino Hueso, L. (2020). La enseñanza digital en serio y el derecho a la educación en tiempos del coronavirus. Revista de Educación y Derecho, 21, 1-29.

Cowling, B. J., Ali, S. T. y Ng T. (2020). Impact assessment of non-pharmaceutical interventions against Covid-19 and influenza in Hong Kong: An observational study. MedRxiv. https://doi.org/10.1101/2020.03.12.20034660.

Dayrit, M. y Mendoza, R. U. (2020). Social Cohesion vs Covid-19. SSRN 3555152, 1-17. https://doi.org/10.2139/ssrn.3555152

De la Cruz Flores, G. (2017). Igualdad y equidad en educación: Retos para una América Latina en transición. Educación, 26(51), 159-178.

Díaz López, C. y Pinto Loría, M. L. (2017). Vulnerabilidad educativa: Un estudio desde el paradigma socio crítico. Praxis Educativa, 21(1), 46-54.

García Gómez, T. (2018). Bases del derecho a la educación: La justicia social y la democracia. Revista Internacional de Educación para la Justicia Social, 7(1), 159-175. https://doi.org/10.15366/riejs2018.7.1.008

Gilbert, M., Pullano, G., Pinotti, F., Valdano, E., Poletto, C., Boëlle, P. Y., D’Ortenzio, E., Yazdanpanah, Y., Eholie, S., Altmann, M., Gutiérrez, B., Kraemer, M. y Colizza, V. (2020). Preparedness and vulnerability of African countries against importations of Covid-19: A modelling study. The Lancet, 395(10227), 871-877.

Giroux, H. (1988). Teachers as intellectuals: Toward a critical pedagogy of learning. Bergin and Garvey.

Jiménez, J. C. (2020). Polémicas educativas en confinamiento. Revista Internacional de Educación para la Justicia Social, 9(3). 
Jones, A., Sergejeff, K., Sherriff, A., Teevan, C. y Veron, P. (2020). The challenge of scaling up the European Union's global response to Covid-19. https://ecdpm. org/publications/challengescaling-upeuropean-union-global-response-covid-19.

Lara Caballero, M. (2017). El derecho a la educación en la medición de pobreza: un análisis complejo. Educación y Humanismo, 19(33), 386-397.

McKibbin, W. J. y Fernando, R. (2020). The global macroeconomic impacts of Covid-19: Seven scenarios. CAMA. Centre for Applied Macroeconomic Analysis, 19, 1-43. https://doi.org/10.2139/ssrn.3547729

Mendieta Izquierdo, G. (2015). Informantes y muestreo en investigación cualitativa. Investigaciones Andina, 17(30), 1148-1150.

Monistrol, O. (2007). El trabajo de campo en investigación cualitativa. Nure Investigación 29, 1-4.

Murillo, F. J. y Hernández, R. (2014). Liderando escuelas justas para la justicia social. Revista Internacional de Educación para la Justicia Social, 3(2), 13-32

Observatorio Social la Caixa. (2020). Objetivo: Paliar la pobreza infantil. Fundación Bancaria "La Caixa”.

ONU. (2017). Informe de los objetivos de desarrollo sostenible. ONU.

Orgilés, M., Morales, A., Delvecchio, E., Mazzeschi, C. y Espada, J. P. (2020). Immediate psychological effects of the Covid-19 quarantine in youth from Italy and Spain. PsyArXiv Preprints. https://doi.org/10.31234/osf.io/5bpfz

Pascual Barrio, B. (2006). Calidad, equidad e indicadores en el sistema educativo español. Pulso. Revista de Educación, 29, 43-58.

Piñuel Raigada, J. L. (2002). Epistemología, metodología y técnicas del análisis de contenido. Sociolinguistic Studies, 3(1), 1-42.

Robledo Martín, J. (2009). Observación participante: Informantes claves y rol del investigador. Nure Investigación, 42, 1-4.

Rundle, A. G., Park, Y., Herbstman, J. B., Kinsey, E. W. y Wang, Y. C. (2020). COVID-19 related school closings and risk of weight gain among children. Obesity.

https://doi.org/10.1002/oby.22813

Sánchez Mendiola, M., Martínez Hernández, A. M., Torres Carrasco, R., Servín, M., Hernández Romo, A. K., Benavides, M., Jaimes Vergara, C. A. y Rendón Cazales, V. J. (2020). Retos educativos durante la pandemia de Covid-19: una encuesta a profesores de la UNAM. Revista Digital Universitaria, en prensa.

Sánchez Silva, M. (2005). La metodología en la investigación cualitativa. Mundo Siglo XXI, 1, $115-$ 118

Simón, C., Barrios, A., Gutiérrez, H. y Muñoz, Y. (2019). Equidad, educación inclusiva y educación para la justicia social. ¿Llevan todos los caminos a la misma meta? Revista Internacional de Educación para la Justicia Social, 8(2), 17-32.

Ting, D. S. W., Carin, L., Dzau, V. y Wong, T. Y. (2020). Digital technology and Covid-19. Nature Medicine, 1-3.

Villafuerte, J., Bello, J., Cevallos, Y. y Bermello J. (2020). Rol de los docentes ante la crisis del Covid-19, una mirada desde el enfoque humano. REFCalE: Revista Electrónica Formación y Calidad Educativa, 8(1), 134-150.

Viner, R. M., Russell, S. J., Croker, H., Packer, J., Ward, J., Stansfield, C., Mytton, O., Bonel, C. y Booy, R. (2020). School closure and management practices during coronavirus outbreaks 
including Covid-19: a rapid systematic review. The Lancet Child \& Adolescent Health. https://doi.org/10.1016/S2352-4642(20)30095-X

Vlachopoulos, D. (2020). Covid-19: Threat or opportunity for online education? Higher Learning Research Communication, 1O(1), 16-19. https://doi.org/10.18870/hlrc.v10i1.1179

Wang, G., Zhang, Y., Zhao, J., Zhang, J. y Jiang, F. (2020). Mitigate the effects of home confinement on children during the Covid-19 outbreak. The Lancet, 395(10228), 945-947.

Zhou, L., Li, F., Wu, S. y Zhou, M. (2020). 'School's out, but class' on', the largest online education in the world today: taking china's practical exploration during the Covid-19 Epidemic prevention and control as an example. Best Evidence of Chinese Education, 4(2), 501-519. http://doi.org/10.2139/ssrn.3555520

\section{Breve CV de los autores}

\section{Jorge Cáceres-Muñoz}

Es profesor ayudante del Departamento de Ciencias de la Educación de la Facultad de Formación del Profesorado de la Universidad de Extremadura. Se doctoró en 2017 por la Universidad de Extremadura recibiendo el premio extraordinario de doctorado. Sus principales líneas de investigación abordan cuestiones relativas tanto a la Teoría como a la Historia de la Educación, narrativas y educación y políticas educativas. Como miembro del Grupo Extremeño de Investigación en Teoría e Historia de la Educación (GEXTHE) ha participado en diferentes proyectos de investigación y coordinado y dirigido seminarios de investigación en educación en temáticas ligadas a la formación del profesorado, la investigación en historia de la educación, y la intervención socioeducativa. ORCID ID: https://orcid.org/0000-0002-2405-9731. Email: jorgecm@unex.es

\section{Antonio Salvador Jiménez Hernández}

Es maestro y doctor en psicopedagogía, docente en la Facultad de Formación del Profesorado de la Universidad de Extremadura, presidente del Consejo Independiente de Protección de la Infancia y director del Laboratorio Internacional de Promoción de Buen Trato y Participación Infantil. Lidera proyectos de cooperación internacional para el desarrollo en el Norte de Marruecos y en América Latina. Autor de veinte libros sobre infancia y educación y de un centenar de otras publicaciones entre ellas capítulos y artículos en revistas científicas. Participa como conferenciante en diversos foros nacionales e internacionales. En 2009 recibió el premio Bandera de Andalucía de Políticas Migratorias por su participación en el proyecto internacional e-Culturas. ORCID ID: https://orcid.org/0000-0002-7805-8929. Email: ajimenez14@unex.es

\section{Miguel Martín-Sánchez}

Es profesor titular de universidad en el área de Teoría e Historia de la Educación de la Universidad de Extremadura-España. Doctor en Educación por la Universidad de Salamanca-España con premio extraordinario, Máster por la Universidad Antonio de Nebrija de Madrid-España y Máster por la Universidad Pablo de Olavide de SevillaEspaña. Ha participado en diversos congresos nacionales e internacionales, y dirigido varios proyectos de investigación y sociales. Sus líneas de investigación se centran en Pedagogía Social, Política de la Educación, Teoría de la Educación e Historia de la Educación. Ha publicado diversos trabajos en revistas científicas nacionales e 
internacionales indexadas en WOS y SCOPUS, y es autor de varios libros en editoriales de reconocido prestigio. ORCID ID: https://orcid.org/O000-0002-9370-1732. Email: miguelmartin@unex.es 